\title{
Efecto del cambio del paisaje en la estructura de la comunidad de abejas sin aguijón (Hymenoptera: Apidae) en Meta, Colombia
}

\author{
Guiomar Nates-Parra ${ }^{1}$, Eliana Palacios ${ }^{2} \&$ Alejandro Parra-H., ${ }^{1,3}$ \\ 1. Laboratorio de Investigaciones en Abejas LABUN, Departamento de Biología, Universidad Nacional de Colombia, \\ Bogotá, Carrera 30 № 45-03, A.A. 14490, Bogotá, Colombia; mgnatesp@unal.edu.co \\ 2. Departamento de Biología. Pontificia Universidad Javeriana; eliana_p_p@yahoo.com \\ 3. varnishpt@yahoo.com
}

Recibido 21-XI-2007. Corregido 07-V-2008. Aceptado 26-V-2008.

\begin{abstract}
Effect of landscape change on the structure of the sting-less bee community (Hymenoptera: Apidae) in Meta, Colombia. Stingless bees represent one of the most diversified components of the natural Apoidea fauna of pollinators in the tropics. They use diverse kinds of substrates and inhabit varied habitats. Some species are typical for some natural either artificial place. The landscape alteration were this group of bees nests, has and important impact on the natural composition of its community structure, fact which is reflected in the nest density. We analyzed the structure composition of the stingless bees' community in three environments in the Colombian llanos piedmont, an important region that represents the transition between Andean ecosystems and a savannah that is seriously threatened by cattle practices. We made systematic samples in secondary forest, agro-ecosystems and urban areas, recording the presence of 204 nests from 11 genera (24 species). The nest density per landscape was heterogeneous and never higher than 16 nests/Ha. We observed two nesting patterns and an effect of sampling criterion on the measured biodiversity. Rev. Biol. Trop. 56 (3): 1295-1308. Epub 2008 September 30 .
\end{abstract}

Key words: Agro-ecosystems, conservation, Colombia, diversity, llanos piedmont, Meliponini, spatial distribution, stingless bees.

Las abejas sin aguijón (Apidae: Meliponini) son parte importante de la comunidad de abejas tropicales. En el Neotrópico son particularmente abundantes y se destacan por su papel como polinizadores de plantas silvestres y cultivadas (Nates-Parra 2005). Nidifican en cavidades de árboles vivos, cavidades subterráneas, termiteros o en cavidades y recipientes artificiales hechos por el hombre (Nates-Parra 2005). Algunas especies nidifican en forma expuesta en ramas de árboles o lianas (Wille y Michener 1973, Roubik 1983). Se conocen varios cientos de especies (Michener 2000) de las cuales cerca de 120 habitan en Colombia (Nates-Parra 2001).

Poco se conoce acerca de la estructura y dinámica de las comunidades de abejas sin aguijón y menos aún acerca de los efectos de las modificaciones en los paisajes naturales sobre estas comunidades; la estructura de las comunidades de abejas sin aguijón puede verse afectada por factores tales como la disponibilidad y la competencia por alimento, sitios de nidificación y depredación (Slaa 2003). La marcada y acelerada modificación de los ambientes naturales por la expansión de la frontera agrícola y ganadera además de la creciente urbanización causa cambios en microclimas, estructura y composición de la vegetación, lo cual influye sobre las comunidades de meliponinos (Kremen et al. 2002). La deforestación afecta tanto la densidad de nidos como la composición de especies y heterogeneidad de la comunidad, sin embargo no se detectan diferencias significativas en diversidad de especies (Slaa 2003). 
El grado en el cual estos factores afectan la estructura de la comunidad es variable por que mientras Hubbell y Johnson (1977) y Eltz et al. (2001) concluyen que factores como la oferta de alimento son más limitantes para la densidad de nidos que la depredación o la disponibilidad de sitios de nidificación, Salmah et al. (1990) determinan que la disponibilidad de sustratos para construir nidos es el factor limitante en el establecimiento de nidos de Trigona minangkabau en Sumatra, puesto que al instalar domicilios artificiales, la población de esa especies de abeja se duplicó.

El número de especies varía en diferentes comunidades locales, desde pocas especies 11-14 en un bosque seco de Costa Rica (Michener 1946, Hubbell y Jonson 1977, respectivamente) hasta 62 especies en la región amazónica (Roubik 1979). De la misma forma la densidad de nidos naturales puede variar desde 1.2 nidos/Ha. (Slaa 2003) hasta 16 nidos/ Ha. (Eltz y Brühl 2002). Sin embargo en hábitats artificiales y muy específicos como los cementerios, la densidad puede incrementarse hasta en 90.9 nidos/Ha. (Nates-Parra et al. 2006).

En Colombia, como en muchos otros países, la transformación del paisaje por cuenta de la deforestación con fines ganaderos alcanza cifras muy altas (600 Ha. anuales, Márquez 1997) y sin embargo existen pocos estudios sobre el efecto que este hecho causa sobre las poblaciones animales y particularmente sobre las comunidades de abeja silvestres (Parra-H y Nates-Parra 2007). La biología en particular de algunas especies de abejas sin aguijón hace que cambios en el medio sean detectables en términos de diversidad de especies sensibles (Brown y Albrecht 2001). Este hecho se ha observado en otros grupos de abejas tropicales (e.g. Parra-H y Nates-Parra 2007) por lo cual es importante analizar la variación de la comunidad de un grupo de abejas que guarda también particularidades biológicas sensibles al medio con el fin de conocer y conservar los ecosistemas naturales.

El piedemonte llanero colombiano es una región importante por que representa la tran- sición entre la cordillera Oriental y la llanura y donde se encuentran zonas de vida como el bosque húmedo tropical (bh-T), bosque muy húmedo tropical (bmh-T), bosque muy húmedo montano bajo (bmh-MB) y zona de transición entre bosque húmedo y seco (Rangel-Ch y Aguilar-P 1995), pero también es una región donde se observa una marcada y acelerada modificación de los ambientes naturales por la expansión de la frontera agrícola y ganadera además por la creciente urbanización (Márquez 1997, 2001). Debido a esto el objetivo de este trabajo fue analizar la variación en la composición de la comunidad de abejas sin aguijón a través de gradientes ambientales y tratar de entender las causas de esta variación para proponer estrategias de conservación

\section{MATERIALES Y MÉTODOS.}

Sitio de estudio. Realizamos el estudio entre diciembre de 2003 y agosto de 2004 en el Piedemonte Llanero $\left(3^{\circ} 55^{\prime}-4^{\circ} 10^{\prime} \mathrm{N}\right.$ y $73^{\circ} 28^{\prime}-73^{\circ} 49^{\prime}$ W) departamento del Meta, Colombia, con altitudes entre los 570 y 660 msnm. Seleccionamos tres paisajes con diferencias en la intensidad de transformación del paisaje original (bosque secundario: $\mathrm{B}$, agroecosistema: A y zona urbana: U). La formación correspondiente a esta región es "bosque húmedo tropical" (bh-T). El comportamiento climático es de régimen monomodal con una época invernal prolongada, desde abril a noviembre. El promedio de precipitación anual es de $2600 \mathrm{~mm}$; la temperatura media es $27^{\circ}$ $\mathrm{C}$ y la humedad relativa varía entre 75 y $90 \%$ (Galvis et al. 1989).

El bosque secundario (B) se localizó en el Jardín Botánico del municipio de Villavicencio, al noroccidente de la ciudad con una extensión de $50 \mathrm{Ha}$. Los estratos dominantes de este paisaje son el arbustivo $(80 \%)$ con especies vegetales distribuidas uniformemente y el subarbóreo $(75 \%)$ con árboles que presentan alturas entre 10 y $12 \mathrm{~m}$. y valores de DAP generalmente menores a 15 $\mathrm{cm}$. Euphoprbiaceae, Rubiaceae, Araliaceae y Melastomataceae son las familias vegetales 
más abundantes en este paisaje. La intervención humana en el lugar es moderada.

El agro-ecosistema (A) seleccionado se localizó en las veredas San José y Orotoy (municipio de Acacías) a $8 \mathrm{Km}$. del casco urbano entre los ríos Acacias y Guamal. Este paisaje se caracteriza por la presencia de fincas ganaderas de grandes extensiones, algunos pocos fragmentos de bosque secundario, cultivos, jardines y huertas. El pastizal es el estrato predominante. Bignonaceae, Caesalpinaceae, Euphorbiaceae, Moraceae y Verbenaceae son las familias vegetales más abundantes del agroecosistema.

El paisaje urbano (U) correspondió a la ciudad de Villavicencio, Capital del Departamento del Meta. La vegetación es de transición entre los bosques de la Cordillera Oriental y el bosque de Sabana (Rangel et al. 1997). En éste paisaje se encuentran parches vegetales de plantas ornamentales o frutales, así como zonas menos intervenidas localizadas a lo largo de los cauces hídricos y en las laderas de la cordillera. Las familias vegetales más abundantes son Moraceae y Cesalpinaceae.

Composición de la comunidad de abejas sin aguijón. En cada paisaje establecimos 25 transectos de $200 \times 10 \mathrm{~m}$ para un total de $5 \mathrm{Ha}$. evaluadas por paisaje. Medimos la densidad (número de nidos por Ha.) y contabilizamos todos los nidos observados en los diferentes tipos de sustratos (naturales y artificiales). Un nido fue considerado como un individuo, por el carácter altamente social de estas abejas. Para cada nido encontrado, registramos datos sobre arquitectura externa tipo de entrada, sustrato de nidificación, altura desde el piso, comportamiento defensivo (según los parámetros establecidos por Wille y Michener (1973) y Nates-Parra y Cepeda (1983) y tomamos muestras de las abejas de cada nido. Recogimos muestras de los árboles donde se encontraron nidos y registramos su tamaño (DAP 130 cm.). Para la determinación taxonómica recurrimos a diferentes claves taxonómicas (Schwarz 1932, 1948, Nates-Parra 2005, Hernández 2004, González 2000) y posterior verificación por especialistas.
Análisis estadístico: Para evaluar el esfuerzo y para determinar la homogeneidad de las muestras, utilizamos la Curva de Coleman (Coleman et al. 1982), junto con la prueba de bondad de ajuste de chi-cuadrado entre las muestras de cada paisaje. Determinamos la riqueza de abejas sin aguijón con base en el número de especies registradas, a la vez que calculamos dos estimadores de riqueza (Jackknife 1 y 2). Comparamos también la riqueza y abundancia de especies de abejas sin aguijón para cada paisaje mediante un análisis de varianza de datos agrupados.

\section{RESULTADOS}

Diversidad y composición de especies. Registramos 204 nidos de 24 especies pertenecientes a 11 géneros de abejas sin aguijón (B: 13 spp., A: 12 spp., U: 15 spp.; Cuadro 1). Las curvas de acumulación de especies, presentaron una tendencia a estabilizarse en el bosque y en el agro-ecosistema (Fig. 1). Esto no se observó para la zona urbana, en la que apreciamos cierta heterogeneidad. Sin embargo, la prueba de bondad de ajuste para la homogeneidad entre los transectos (chi-cuadrado), no estableció diferencias, por lo tanto consideramos que las unidades muestreales fueron homogéneas $\left(\mathrm{X}^{2}<36.4\right)$. Los estimadores de riqueza mostraron que para el bosque y el agro-ecosistema los datos se ajustan a la escala de valores observada (12-15 spp.). Esto indica una eficiencia del muestreo con cerca del $100 \%$ de especies encontradas para el estimador Jackknife 2 y en un $82 \%$ para Jackknife 1 . El análisis de varianza mostró que la riqueza y abundancia de la comunidad de abejas sin aguijón es significativamente distinta en los tres paisajes muestrea$\operatorname{dos}(\mathrm{F}=11.7484, \mathrm{p}<0.0001, \alpha=0.05)$.

Alrededor del $50 \%$ de las especies fueron similares entre los paisajes. El $12.5 \%$ de las especies (Tetragona perangulata, Trigona angustula, T. amalthea) están presentes en los tres paisajes, mientras que el $45.3 \%$, se encuentra solo en uno: $25 \%$ especies únicas del bosque, $8.3 \%$ exclusivas del agro-ecosistema y $12.5 \%$ se encontraron solamente en la zona 
CUADRO 1

Abundancia relativa de las especies de abejas sin aguijón encontrada en diferentes paisajes en el piedemonte llanero

TABLE 1

Relative abundance of stingless bees in several landscapes of the Colombian piedmont

Especie

Cephalotrigona capitata

Geotrigona fulvohirta

Lestrimelitta limao

Melipona compressipes

Melipona eburnea

Melipona favosa

Melipona fuliginosa

Nannotrigona melanocera

Oxytrigona mediorufa

Partamona aff. testacea

Partamona peckolti

Partamona testacea

Plebeia (Plebeia) frontalis

Plebeia (Scaura) longula

Scaptotrigona barrocoloradensis

Scaptotrigona limae

Scaptotrigona nigella

Tetragona perangulata

Trigona (Tetragonisca) angustula

Trigona (Trigona) amalthea

Trigona (Trigona) fulviventris

Trigona (Trigona) fuscipennis

Trigona (Trigona) spinipes

Trigona (Trigona) williana
Bosque

$\%$

2.1 2.1

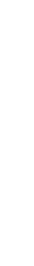

10.6

17.0

4.2

17.0

4.2

4.2

14.8

6.3

8.5

4.2

4.2

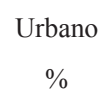

1.3

0

2.6

1.3

16.8

3.9

1.3

5.1

9.0

2.6

10.3

9.0

31.1

2.6

1.3

2.5

1.2

Agro-ecosistema
$\%$

2.5

12.5

1.2

41.0

5

5

5

11.3

5

1.3 urbana. Plebeia (Scaura) longula y Partamona testacea fueron las especies más abundantes del bosque; Nannotrigona melanocera del agroecosistema y T. angustula de la zona urbana.

Sustrato de nidificación. Las abejas sin aguijón se encontraron nidificando en árboles vivos, muertos, termiteros, suelo, muro y otras cavidades artificiales. 28 especies arbóreas, pertenecientes a 19 familias vegetales son utilizadas por las abejas sin aguijón para hacer sus nidos (Cuadro 2). Los árboles vivos fueron el sustrato preferido para nidificar $(43.6 \%$ de nidos y $67 \%$ de especies), principalmente en el tronco $(73 \%)$, y pocas veces en la base (14\%). El 62\% de los nidos encontrados en árboles vivos se concentró en unas pocas especies: varias especies (cuatro) de caucho 

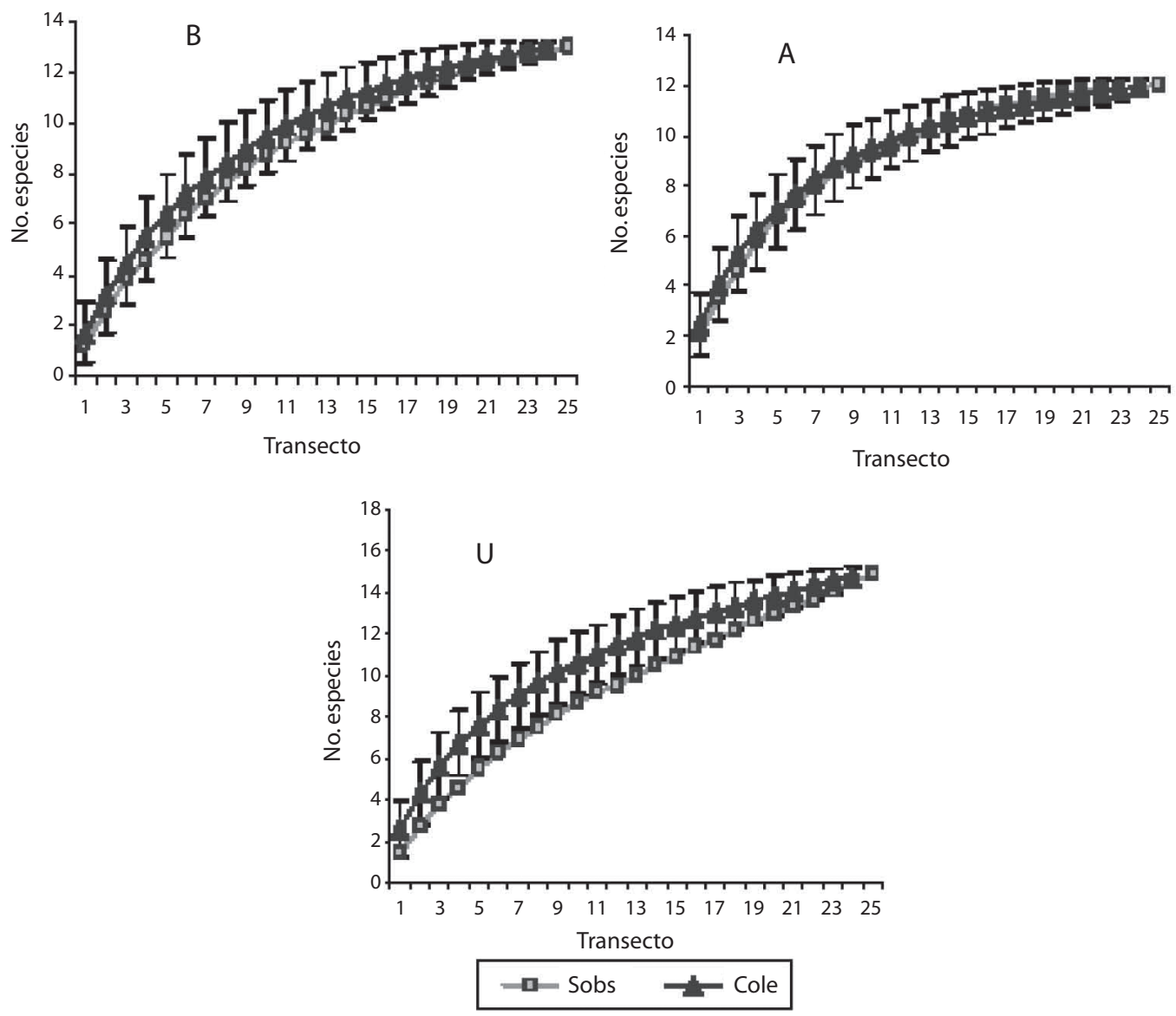

Fig. 1. Curvas de acumulación de especies esperadas [Coleman (Cole), con su respectiva desviación estándar] y observadas (Sobs), en 25 transectos para la comunidad de abejas sin aguijón en el bosque (B), agro-ecosistema (A) y zona urbana (U) del Piedemonte llanero.

Fig. 1. Cummulative curves of expected species [Coleman (Cole), with standard deviation] and observed (Sobs), in 25 transects for the stingless bee community in forest (B), agro-ecosystem (A) and urban zone (U) in the Colombian piedmont.

(Ficus spp.), nocuito (Vitex orinocense), guayabo (Psidium guajava) y Eucalipto (Eucalyptus sp.). Aproximadamente el $50 \%$ de las especies de meliponinos nidifica en esas siete especies vegetales.

V. orinocense, P. guajava, Eucalyptus sp., Talauma sp., Cousapoa villosa, Cesalpine sp. y Socratea exorhiza son especies que ofrecen cavidades a más de tres especies de abejas sin aguijón, mientras que especies como Ochroma sp., Croton sp. y Cinchona sp. ofrecen sitio para nidificación solamente a $T$. perangulata; Ceiba sp. lo hace solamente para $T$. spinipes. Encontramos cinco especies (20.3\%) nidificando en árboles vivos a 10 o mas metros de altura.

El bosque presentó la mayor densidad de árboles con un $\mathrm{DAP}>10 \mathrm{~cm}$. que potencialmente servían como sitio de nidificación (bosque: 99.2 árboles $/ \mathrm{km}^{2}$; agro-ecosistema: 60.5 árboles $/ \mathrm{km}^{2}$ : zona urbana: 49 árboles $/ \mathrm{km}^{2}$ ). El DAP de los árboles utilizados para nidificación, en 
CUADRO 2

Densidad de nidos por especie según el sustrato y el tipo de paisaje

TABLE 2

Nest density per species, substrate and landscape type

\begin{tabular}{|c|c|c|c|}
\hline Especie & Paisaje & Nidos/Ha. & Sustrato \\
\hline Cephalotrigona capitata & Bosque & 0.2 & Eucalyptus sp. \\
\hline Geotrigona fulvohirta & Urbano & 0.2 & Suelo \\
\hline Lestrimelitta limao & Conservado & 0.2 & Termitero \\
\hline Melipona compressipes & Agro-ecosistema & 0.4 & Psidium guajava \\
\hline Melipona eburnea & Agro-ecosistema, Urbano & $0.4-2$ & Vitex orinocense, Cavidad artificial \\
\hline Melipona favosa & Urbano & 0.2 & Artificial \\
\hline $\begin{array}{l}\text { Melipona fuliginosa } \\
\text { Nannotrigona melanocera }\end{array}$ & $\begin{array}{l}\text { Agro-ecosistema, } \\
\text { Urbano }\end{array}$ & $0.2-2.6$ & $\begin{array}{l}\text { Tronco muerto, Acacia sp., Cesalpine } \\
\text { sp., Psidium guajava, Sapium sp., } \\
\text { Talauma sp., Vitex orinocense }\end{array}$ \\
\hline Oxytrigona mediorufa & Agro-ecosistema, Urbano & $0.6-0.8$ & $\begin{array}{l}\text { Cedrela sp., Psidium guajava, Tronco } \\
\text { muerto }\end{array}$ \\
\hline Plebeia (Plebeia) frontalis & Bosque & 0.4 & Cousapoa villosa \\
\hline Plebeia (Scaura) longula & Bosque, Urbano & $1.4-1.6$ & Eucalyptus sp. Termitero \\
\hline Partamona aff. testacea & Bosque & 1 & Cavidad artificial \\
\hline Partamona peckolti & $\begin{array}{l}\text { Agro-ecosistema, } \\
\text { Urbano }\end{array}$ & $0.2-1.2$ & $\begin{array}{l}\text { Talauma sp., Vitex orinocense, Cavidad } \\
\text { artificial }\end{array}$ \\
\hline Partamona testacea & Bosque, Urbano & $0.6-1.6$ & Suelo, Cavidad artificial \\
\hline Scaptotrigona barrocoloradensis & Bosque & 0.4 & Socratea exorhiza \\
\hline Scaptotrigona limae & Agro-ecosistema, Urbano & $0.4-0.8$ & Tronco muerto, Cavidad artificial \\
\hline Scaptotrigona nigella & Urbano & 1.6 & Ficus ficus \\
\hline Trigona (Tetragonisca) angustula & $\begin{array}{l}\text { Agro-ecosistema, } \\
\text { Bosque, Urbano }\end{array}$ & $1.4-4.8$ & $\begin{array}{l}\text { Ficus sp., Vitex orinocense, Cesalpine } \\
\text { sp., Dendropanax sp., Erythrina } \mathrm{sp} . \\
\text { Eucalyptus sp., Ficus ficus, Tabebuia } \\
\text { sp., Cavidad artificial }\end{array}$ \\
\hline Tetragona perangulata & $\begin{array}{l}\text { Agro-ecosistema, } \\
\text { Bosque, } \\
\text { Urbano }\end{array}$ & $0.4-1.4$ & Croton sp., Cavidad artificial \\
\hline Trigona (Trigona) amalthea & $\begin{array}{l}\text { Agro-ecosistema, } \\
\text { Bosque, Urbano }\end{array}$ & $0.4-0.8$ & $\begin{array}{l}\text { Talauma sp., Vitex orinocense, Cedrela } \\
\text { sp., Eucalyptus sp., Ficus ficus, Tronco } \\
\text { muerto }\end{array}$ \\
\hline Trigona (Trigona) fulviventris & Bosque, Urbano & $0.2-0.8$ & $\begin{array}{l}\text { Suelo } \\
\text { Tronco muerto }\end{array}$ \\
\hline Trigona (Trigona) fuscipennis & Agro-ecosistema, Bosque & 0.4 & $\begin{array}{l}\text { Cavidad artificial, Ceiba petandra, } \\
\text { Ficus sp., Socratea exorhiza, Termitero }\end{array}$ \\
\hline Trigona (Trigona) spinipes & Agro-ecosistema & 0.2 & Ceiba sp. \\
\hline Trigona (Trigona) williana & Bosque, Urbano & $0.2-0.4$ & Dendropanax sp., Ficus sp. \\
\hline
\end{tabular}


todos los paisajes osciló entre $9.14 \mathrm{~cm}$ y 142.4 $\mathrm{cm}$. Para facilitar el análisis establecimos cuatro categorías según valores de DAP. El tamaño más utilizado estuvo en las categorías 2 y 3 , entre 20 a $60 \mathrm{~cm}(62.1 \%$ de todos los registros y $84.2 \%$ de las especies, Cuadro 3; Fig. 2).

$T$. angustula y $T$. perangulata fueron las especies más plásticas ya que las encontramos nidificando en todas las categorías de DAP, mientras que especies como Cephalotrigona capitata, Melipona compressipes, Lestrimelitta limao, $P$. frontalis tuvieron sus nidos solamente en una de las categorías de DAP (Cuadro 3).

C. capitata, N. melanocera, T. angustula, $M$. compressipes y $T$. perangulata nidificaron en árboles con los mayores DAP (mayores de $100 \mathrm{~cm}$.). La mayor parte de los árboles comprendidos en esta escala (categoría 4) se ubicaron en el paisaje rural. La mayor cantidad de nidos encontrados en árboles en el bosque correspondió a las categorías 1 y 2 mientras que en la zona urbana los nidos se encontraron en mayor cantidad en las categorías 2 y 3 (Cuadro 3).
Especies arbóreas abundantes en el paisaje y con cavidades disponibles se utilizan con mayor frecuencias como por ejemplo F. ficus, $V$. orinocense, P. guajava, Eucalyptus sp. Los muros fueron el segundo sustrato mas importante $(26.4 \%$ de los nidos) ocupados especialmente por T. angustula y N. melanocera y los termiteros (Nasutemittes sp.) ocuparon el tercer lugar en preferencia $(9.31 \%)$ particularmente por $P$. longula y T. fuscipennis. La mayor parte de los nidos encontrados estaban ocultos dentro de una cavidad (95\%).

El suelo, aunque en menor proporción, fue otro de los sustratos donde encontramos nidos particularmente de Geotrigona fulvohirta y $P$. testacea, esta última en terrenos arcillosos y asociada con termites. T. angustula nidifica en diferentes tipos de sustrato, mientras que especies como M. compressipes, P. testacea o G. fulvohirta nidifican en solo una clase de sustrato.

Distribución espacial de las especies y de los nidos. En la zona de estudio encontramos

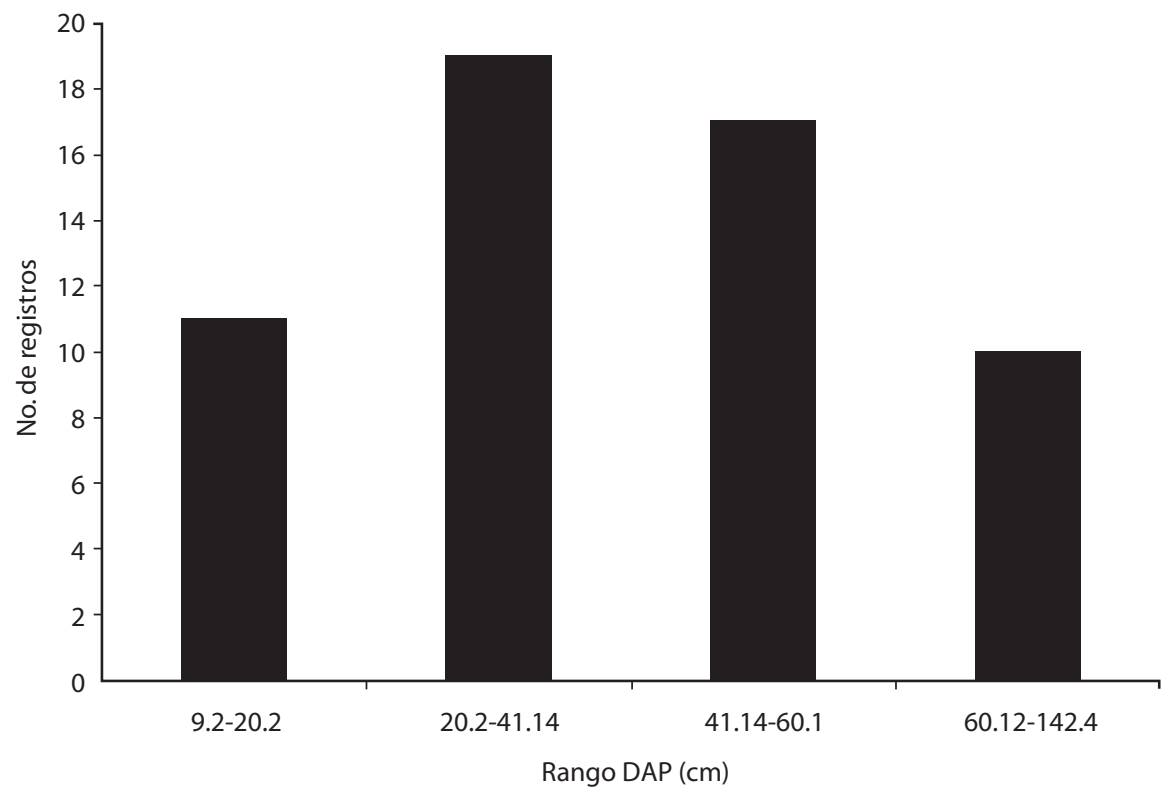

Fig. 2. Número de nidos registrados por categoría de DAP.

Fig. 2. Number of nests per DBH class. 
CUADRO 3

Categorias de DAP (cm.) por paisaje (B: Bosque, U: Urbano, R: Agro-ecosistema) utilizados por las especies de abejas sin aguijón como sustrato de nidificación

TABLE 3

DBH categories (cm) by landscape (B: forest, U: urban, R: Agro-ecosystem) used by stingless bees to nest

\begin{tabular}{|c|c|c|c|c|c|c|}
\hline & 1 & 2 & 3 & 4 & Total & Paisaje \\
\hline Especies & $9.2-20.2$ & $20.2-41.14$ & 41.14- 60.1 & $60.12-42.4$ & & \\
\hline Cephalotrigona capitata & & & & 1 & 1 & B \\
\hline Lestrimelitta limao & & & 1 & & 1 & B \\
\hline Melipona compressipes & & & & 1 & 1 & $\mathrm{R}$ \\
\hline Melipona eburnea & 1 & 1 & & & 2 & $\mathrm{R}$ \\
\hline Nannotrigona melanocera & & 3 & 1 & 3 & 7 & $\mathrm{U} / \mathrm{R}$ \\
\hline Plebeia (Plebeia) frontalis & 1 & & & & 1 & $\mathrm{U}$ \\
\hline Scaptotrigona barrocoloradensis & & 2 & & & 2 & B \\
\hline Scaptotrigona nigella & & & 8 & & 8 & $\mathrm{U}$ \\
\hline Scaptotrigona limae & & & 1 & 1 & 2 & $\mathrm{R} / \mathrm{U}$ \\
\hline Plebeia (Scaura) longula & 2 & 2 & & & 4 & $\mathrm{~B} / \mathrm{U}$ \\
\hline Tetragona perangulata & 3 & 2 & 1 & 3 & 9 & $\mathrm{~B} / \mathrm{U} / \mathrm{R}$ \\
\hline Trigona (Trigona) amalthea & & 1 & & & 1 & $\mathrm{~B} / \mathrm{U} / \mathrm{R}$ \\
\hline Trigona (Tetragonisca) angustula & 2 & 3 & 3 & 3 & 11 & $\mathrm{~B} / \mathrm{U} / \mathrm{R}$ \\
\hline Trigona (Trigona) fulviventris & & 1 & & & 1 & $\mathrm{~B} / \mathrm{U}$ \\
\hline Trigona (Trigona) fuscipennis & 2 & & 1 & & 3 & $\mathrm{~B} / \mathrm{U}$ \\
\hline Trigona (Trigona) spinipes & & 1 & & & 1 & $\mathrm{R}$ \\
\hline Trigona (Trigona) williana & & 1 & 1 & & 2 & $\mathrm{~B} / \mathrm{U}$ \\
\hline
\end{tabular}

abejas sin aguijón nidificando de forma agrupa$\mathrm{da}$, uniforme $\mathrm{o}$ al azar. Algunas especies mostraron agrupaciones de sus nidos: $N$. melanocera, especie dominante en el agro-ecosistema con nidos localizados a pocos metros de distancia ( $\sim 10$ m. máximo) uno de otro o sobre el mismo sustrato, $P$. testacea y $P$. aff. testacea nidifican generalmente agrupadas en un mismo banco de arena, de dos hasta cuatro nidos, $P$. longula se encontró hasta con siete nidos en un mismo termitero. Otras especies también presentaron distribución agrupada, pero menos evidente en la observación en campo. En el agro-ecosistema y en la zona urbana, la distribución agrupada se presentó a manera de agregaciones de nidos de dos o tres especies sobre un mismo sustrato.
La agregación de nidos también se da en especies con abundancias medias, como P. peckolti (hasta tres nidos por sustrato). Ocho nidos de Scaptotrigona nigella se encontraron en un mismo árbol mientras que M. eburnea presentó nidificación al azar.

La distancia entre los nidos de las diferentes especies fue variable: las especies del bosque con distribución agrupada, pocas veces presentaban otras especies nidificando a distancias menores de $10 \mathrm{~m}$., sin embargo, en la zona urbana y en el agro-ecosistema se presentaron agregaciones de dos o tres especies diferentes. Especies con nidos individuales generalmente tenían nidos de otras especies muy cerca. $T$. angustula se distribuyó de manera uniforme 
en el bosque, donde encontramos pocos nidos, mientras que en el agro-ecosistema y la zona urbana (muchos nidos) se presentó de manera agrupada.

Densidad de nidos y abundancia de especies. El agro-ecosistema y la zona urbana presentaron mayores valores en la densidad y número de nidos y muy similares entre ellos (agro-ecosistema: 16 nidos/Ha y 80 nidos; urbano: 15.4 nidos/Ha y 77 nidos), mientras que en el bosque fue menor ( 9.4 nidos/Ha y 47 nidos). En cuanto a las especies de abejas, $N$. melanocera y $T$. angustula fueron no solo las especies mas abundantes, sino las que presentaron los valores mas altos de densidad, en todos los tres paisajes (3.1 nidos/Ha y 2.7 nidos/Ha respectivamente). Las densidades de las demás especies no pasaron de un nido por hectárea en todos los paisajes (Cuadro 4).

\section{DISCUSIÓN}

Diversidad y composición de especies. La estructura de la comunidad de abejas sin aguijón fue significativamente diferente aun cuando la riqueza no varió de manera importante en los tres paisajes muestreados. El hecho que algunas especies presenten una preferencia por un paisaje como es el caso de $P$. testacea para la cual el $96 \%$ del total de sus nidos fueron encontrados en áreas conservadas o N. melanocera con el $71 \%$ de sus nidos en áreas rurales (agro-ecosistema), permite asumir varias razones por la cual una comunidad de abejas sin aguijón puede ser homogénea en cuanto a riqueza pero disímil en la estructura.

Los paisajes que presentaban alta densidad de árboles, menor transformación del paisaje original (bosque) y que además, ofrecían potencialmente sitios de nidificación (naturales o artificiales: urbano), presentaron mayores valores de diversidad. De esta manera, es probable que la disponibilidad de sitios naturales para nidificar (árboles con $\mathrm{DAP}>10 \mathrm{~cm}$.) y de alimento, puedan tener alguna relación con la diversidad y uniformidad de las especies. Aunque se esperaba que estos valores fueran menores en la zona urbana, este paisaje se caracterizó por la presencia continua de flores en jardines, cavidades hechas por el hombre y en algunos sectores, de árboles. Por el contrario, sitios fuertemente transformados (agro-ecosistema: baja densidad de árboles), presentaron valores de diversidad menores.

En sitios altamente intervenidos, puede limitarse la dispersión y generar competencia entre especies, favoreciendo a unas pocas las cuales llegan a ser muy abundantes (Hubell y Johnson 1977, Eltz y Brühl 2002). Esto puede corroborarse cuando se compara la densidad de nidos de meliponinos en lugares donde hay muchos sitios de nidificación disponibles (tumbas en los cementerios, 90 nidos/Ha NatesParra et al 2006), con la densidad en sitios conservados ( 9.4 nidos/Ha.) o zonas rurales (16 nidos/Ha.).

Las especies bien representadas en la muestra para cada uno de los paisajes tendrían mayor capacidad de uso de distintos recursos, mientras que existirían otras más dependientes del tipo de oferta y serían especies restringidas a ambientes conservados (C. capitata, M. fuliginosa, $S$. barrocoloradensis, $T$. williana).

Algunos investigadores han planteado, que la presencia de abejas sin aguijón en paisajes perturbados, es favorecida por no existir depredadores o enemigos naturales en estos lugares (Eltz y Brühl 2002). Sin embargo, también establecen que la diversidad de meliponinos en bosques sería mayor, si no existiera la limitación en la observación más detallada del dosel por su elevada altura (Eltz et al. 2001, Roubik 1989).

Sustratos de nidificación. Si bien no observamos preferencias por alguna especie vegetal en particular para nidificar, si hubo una tendencia muy clara hacia un determinado DAP (Fig. 2). Árboles muy frecuentes y con DAP entre 20 y $60 \mathrm{~cm}$ fueron los mas aprovechados para nidificación; estas preferencias coinciden con aquellas registradas en Bolivia para $T$. angustula $(37.4 \mathrm{~cm})$ y M. rufiventris $(42.4 \mathrm{~cm})$ (Copa-Alvaro 2004). Entre los árboles mas utilizados en nidificación se destacan especies del género Ficus (Moraceae) confirmando las 
CUADRO 4

Densidad de nidos de abejas sin aguijón en los tres paisajes estudiados

TABLE 4

Density of stingless bee nests in the three studied landscapes

$\begin{array}{ccc}\text { Bosque } & \text { Urbano } & \text { Agro-ecosistema } \\ 5 \text { ha } & 5 \text { ha } & 5 \text { ha }\end{array}$

\begin{tabular}{|c|c|c|c|c|c|c|}
\hline Especies & No. nidos & $\mathrm{D}$ & \# & $\mathrm{D}$ & \# & \\
\hline Cephalotrigona capitata & 1 & 0.2 & 0 & 0 & 0 & \\
\hline Geotrigona fulvohirta & 0 & 0 & 1 & 0.2 & 0 & \\
\hline Lestrimelitta limao & 1 & 0.2 & 0 & 0 & 0 & \\
\hline Melipona compressipes & 0 & 0 & 0 & 0 & 2 & \\
\hline Melipona eburnea & 0 & 0 & 2 & 0.4 & 10 & \\
\hline Melipona favosa & 0 & 0 & 1 & 0.2 & 0 & \\
\hline Melipona fuliginosa & 0 & 0 & 0 & 0 & 1 & \\
\hline Nannotrigona melanocera & 0 & 0 & 13 & 2.6 & 33 & \\
\hline Oxytrigona mediorufa & 0 & 0 & 3 & 0.6 & 4 & \\
\hline Partamona aff. testacea & 5 & 1 & 0 & 0 & 0 & \\
\hline Partamona peckolti & 0 & 0 & 1 & 0.2 & 6 & \\
\hline Partamona testacea & 8 & 1.6 & 4 & 0.8 & 0 & \\
\hline Plebeia (Plebeia) frontalis & 2 & 0.4 & 0 & 0 & 0 & \\
\hline Plebeia (Scaura)longula & 8 & 1.6 & 7 & 1.4 & 0 & \\
\hline Scaptotrigona barrocoloradensis & 2 & 0.4 & 0 & 0 & 0 & \\
\hline Scaptotrigona limae & 0 & 0 & 2 & 0.4 & 4 & \\
\hline Scaptotrigona nigella & 0 & 0 & 8 & 1.6 & 0 & \\
\hline Tetragona perangulata & 2 & 0.4 & 7 & 1.4 & 4 & \\
\hline Trigona (Tetragonisca) angustula & 7 & 1.4 & 24 & 4.8 & 9 & \\
\hline Trigona (Trigona) amalthea & 3 & 0.6 & 2 & 0.4 & 4 & \\
\hline Trigona (Trigona) fulviventris & 4 & 0.8 & 1 & 0.2 & 0 & \\
\hline Trigona (Trigona) fuscipennis & 2 & 0.4 & 0 & 0 & 2 & \\
\hline Trigona (Trigona) spinipes & 0 & 0 & 0 & 0 & 1 & \\
\hline Trigona (Trigona) williana & 2 & 0.4 & 1 & 0.2 & 0 & \\
\hline Total & \multicolumn{2}{|c|}{47} & \multicolumn{2}{|c|}{77} & \multicolumn{2}{|c|}{80} \\
\hline Densidad total & \multicolumn{2}{|c|}{$9.4 \mathrm{n} / \mathrm{ha}$} & \multicolumn{2}{|c|}{$15.4 \mathrm{n} / \mathrm{ha}$} & & \\
\hline
\end{tabular}


observaciones de Aguilar (1999) para Costa Rica donde señala varias especies del género como sustrato de nidificación importante de S. pectoralis, M. beechei y $T$. angustula. $V$. orinocense es una especie maderable, nativa (Bustamante 1999) utilizada como sombrío para ganado y que además representa una buena fuente de polen para las abejas. P. guajava además proporciona polen, especialmente a las especies del género Melipona y Eucalyptus sp. árbol maderable, también proporciona polen.

Las especies más abundantes en los paisajes intervenidos, pueden hacer más de dos nidos por sustrato (12 nidos sobre un muro de un área de $6 \mathrm{~m}^{2}$ ), dando lugar a una distribución espacial principalmente agrupada y por parte de algunas especies en estos paisajes transformados, mientras que en el bosque, la mayor parte de las especies se distribuyeron de una manera uniforme.

De igual manera, en paisajes intervenidos, generalmente se presentan una gran variedad de sustratos, tanto naturales (árboles, suelo, termiteros) como artificiales (hechos por el hombre) para nidificar, mientras que el bosque tan solo ofrece cavidades naturales. Por lo tanto, algunas especies se ven claramente favorecidas por su adaptación para nidificar en cualquier tipo de sustrato. Sin embargo, el sustrato preferido y por lo tanto limitante para la mayor parte de especies son las cavidades arbóreas (Roubik 1979, 1983, 1989, Eltz et al. 2001, Slaa 2003)

Densidad de nidos y abundancia de especies. Algunas especies presentaron ciertas variaciones en cuanto a densidad y abundancia en relación con el cambio en el paisaje. Por ejemplo, el número de nidos de T. angustula y T. perangulata, aumentó en paisajes perturbados, mientras que para T. amalthea, disminuyó. Esta última especie nidifica preferiblemente en árboles con un gran DAP (Hernández 2004, Nates-Parra y Parra-H 2006), lo que puede mostrar su notable reducción en sitios transformados. Especies como C. capitata y $S$. barrocoloradensis, encontradas en el bosque, solo presentan registros conocidos, además pocos, en este tipo de ecosistemas (NatesParra 1983).

Los valores de densidad aquí obtenidos muestran que los ambientes intervenidos proporcionan diversos tipos de cavidades que son aprovechadas por las abejas sin aguijón para hacer sus nidos. Hubbell y Johnson (1977) y Copa-Alvaro (2004) mencionan que la preferencia por sitios de nidificación esta dada más por disponibilidad de cavidades que por presencia de especies particulares de árboles. Además es posible que en estos ambientes intervenidos las abejas encuentren abundantes fuentes alimenticias proporcionadas por jardines y parques de las ciudades o de los sectores rurales. Especies como T. angustula y N. melanocera son las que mas utilizan este tipo de cavidades y por eso la densidad de sus nidos es muy elevada (Cuadro 4).

Nuestros datos son semejantes a los reportados por algunos investigadores en diferentes regiones tropicales (0-16 nidos/ha: Michener 1946, Roubik 1983, 1996, Salmah et al. 1990, Nagamitsu y Inoue 1997, Eltz et al. 2002, Slaa 2003) y no van mas allá de 16 nidos/Ha. Esto podría significar que la capacidad de carga de un ecosistema no alcanza a sostener más de 16 nidos/Ha.

A nivel de especie la densidad de nidos se comporta de manera similar a la densidad de nidos por paisaje, es decir en el ambiente conservado la disponibilidad de nidificación por especie es de 0.72 nidos/Ha, en el urbano es de 1.03 nidos/Ha y en el agro-ecosistema es de 1.33 nidos/Ha. Sin embargo la distribución no es equitativa y la mayoría de las especies de cada comunidad presenta densidades muy por debajo del promedio y unas pocas tienen densidades considerablemente mayores a este promedio. Nidos de $P$. testacea son especialmente abundantes en bosques por que es el único ambiente donde existe este sustrato (bancos de arena asociados a termiteros). $T$. angustula por su gran adaptabilidad a cavidades artificiales y su capacidad de forrajear en diversas de plantas desde ornamentales hasta rastrojos y árboles de gran porte, ocupa con mayor frecuencia los locales ofrecidos por los 
ambientes urbanos. Además, su dispersión a cortas distancias favorece el establecimiento de muchos nidos en áreas reducidas. Lo mismo sucede con $N$. melanocera pero con una marcada preferencia por agro-ecosistemas.

La estructura de la comunidad de abejas sin aguijón en los distintos paisajes estaría dada por un equilibrio en la capacidad por obtener y competir por los distintos recursos incluyendo sustratos de nidificación. La capacidad en términos de éxito, sería dependiente de la plasticidad en que alguna $u$ otra especie obtiene los distintos recursos además de la manera y donde nidifica, por lo cual, al transformarse progresivamente la calidad del medio, aquellas especies más plásticas empezarían a verse beneficiadas. Sin embargo este hecho no se ve reflejado en la aparición de algunas especies típicas si no en el cambio de la estructura de la comunidad en sí.

Dentro de esta comunidad cada especie $X_{n}$ de abejas usará $Y_{n}$ recursos dependiendo de la amplitud de nicho de cada especie. Cuando una especie $\mathrm{X}_{1}$ que usa un recurso $\mathrm{Y}_{1}$ (dentro de su espectro de polilectia y preferencias por locales de nidificación) desaparece del hábitat donde está establecida la comunidad, este recurso $\mathrm{Y}_{1}$ podría ser utilizado por otras especies de la misma comunidad dependiendo de dicha plasticidad. Sin embargo este recurso también podría ser utilizado por especies de otros hábitats cercanos de manera que la comunidad en sí no cambiaría de manera importante en su diversidad.

El cambio de los paisajes naturales no necesariamente implica una disminución en la diversidad de las comunidades que los habitan. Por el contrario las especies de abejas silvestres, encuentran sitios adecuados para su nidificación y manutención en áreas intervenidas (Shepherd 2003). Pero sin embargo, es importante reasaltar que cada paisaje se caracteriza por una composición de especies con densidades de nidos particulares.

Es de resaltar que si bien la curva de acumulación y los estimadores de riqueza indican que el esfuerzo fue eficiente, en este trabajo se reporta un total de 24 especies de abejas sin aguijón, pero para las mismas localidad y en el mismo período de recolección de este trabajo, muestreos con redes entomológicas registraron hasta cerca de 38 especies (Nates-Parra, datos sin publicar). Como se ha observado con otros grupos de abejas, la estimación de la riqueza, abundancia y en general diversidad de este grupo de organismos depende de las características biológicas del grupo en particular (e.g. Parra-H y Nates-Parra 2007). Para entender la dinámica de una comunidad debe buscarse parámetros e indicadores diseñados para cada grupo, por lo cual es necesario estudios puntuales de floras, unidades fisiográficas y variables ambientales, entre otros, en relación a la biología del grupo. De otra forma, estimadores ampliamente usados pueden no ser realmente útiles.

\section{AGRADECIMIENTOS}

Los autores agradecen a los integrantes del LABUN (D. Vélez, A. Rodríguez, M.I. Gómez y P. Baquero) por su colaboración en las fases iniciales del proyecto. A C. Rivera y B. Mantilla por su apoyo, V.H. González y M.A. Bonilla por sus sugerencias y a J.M.F. Camargo por la identificación de algunas especies. A la comunidad de la vereda de S. José de Acacías y a las directivas del Jardín Botánico de Villavicencio por su colaboración. A la Estación de Biología Tropical Roberto Franco de la Universidad Nacional (Villavicencio) por el apoyo logístico. A la Universidad Nacional de Colombia, Facultad de Ciencias, Departamento de Biología (sede Bogotá) por su apoyo y a la División de Investigaciones de Bogotá (DIB) de la UN por la financiación del proyecto Cría y manejo de abejas sin aguijón en el Piedemonte llanero a G. Nates-Parra (convocatoria Prometeo No.201010024542003).

\section{RESUMEN}

Las abejas sin aguijón son unos de los polinizadores naturales más diversos de la fauna Apoidea en los trópicos. Nidifican en diversos sustratos y distintos ambientes, existiendo algunas especies típicas de lugares naturales o artificiales. La alteración del medio donde nidifican tiene un importante impacto sobre la composición natural de su estructura, hecho que se ve reflejado también en 
la densidad de nidos. Analizamos la composición de la estructura de la comunidad de abejas sin aguijón en tres paisajes del piedemonte llanero colombiano, una región importante por representar la transición entre ambientes andinos y de sabana (seriamente amenazada por la ganadería intensiva). Realizamos muestreos sistemáticos en bosque secundario, agro-ecosistema y zona urbana; registramos la presencia de 204 nidos de 11 géneros representados por 24 especies. La riqueza de abejas sin aguijón fue similar, aun cuando hubo diferencias significativas en la estructura de la comunidad en los tres paisajes. La densidad de nidos por paisaje fue heterogénea y nunca mayor de 16 nidos/Ha. Observamos dos patrones de nidificación y registramos diferente número de especies en la misma zona utilizando otro criterio de muestreo.

Palabras clave: Abejas sin aguijón, agro-ecosistemas, Colombia, distribución espacial, diversidad, Meliponini, Piedemonte llanero.

\section{REFERENCIAS}

Copa-Alvaro, M. 2004. Patrones de nidificación de Trigona (Tetragonisca) angustula y Melipona rufiventris (Hymenoptera: Meliponini) en el norte de La Paz, Bolivia. Ecol. Apl. 3: 82-86.

Eltz, T., C. Brühl, S. Van der Kaars, V.K. Chey, K.E. Linsenmair. 2001. Pollen foraging and resource partioning of stingless bees in relation to flowering dynamics in a Southeast Asian tropical rainforest. Insectes. Soc. 48: 273-279.

Eltz, T. \& C. Brühl. 2002. Ecological evaluation of forest management using leaf-litter ants and stingless bees as indicators. Deutsche Gesellschaft für. Technische Zusammenarbeit (GTZ) GmbH. Begleitprogramm Tropenökologie (TÖB). Tropical Ecology Support Program. Federal Republic of Germany.

Fernández, F. 1995. La diversidad de los Himenópteros en Colombia, p. 373-442. En: Rangel, J.O. (ed.). Colombia diversidad biótica 1. Universidad Nacional de Colombia e Inderena, Santafé de Bogotá. Colombia.

Galvis, G., J.I. Mojica \& F. Rodríguez. 1989. Estudio ecológico de una laguna de desborde del Río Metica. Fondo FENCOLOMBIA. Universidad Nacional de Colombia, Bogotá, Colombia.

González, V.H. 2000. El género Oxytrigona en Colombia. Trabajo de Grado. Biología, Facultad de Ciencias, Universidad Nacional de Colombia, Bogotá, Colombia.

Hernández, J. 2004 El subgénero Trigona s. str. Jurine 1808 (Hymenoptera: Apidae: Meliponini) en Colombia.
Trabajo de Grado. Biología, Facultad de Ciencias, Universidad Nacional de Colombia, Bogotá,

Hubell, S.P. \& L.K. Johnson. 1977. Competition and nest spacing in a tropical stingless bee community. Ecology 58: 949-963.

Kremen, C., N.M. Williams, \& R.W. Thorp. 2002. Crop pollination from native bees at risk from agricultural intensification. Pnas: 16812-16816.

Márquez, G. 1997. Ecosistemas y biodiversidad en Colombia. Memorias del seminario de "Biodiversidad y manejo de fauna silvestre". Instituto de estudios ambientales Universidad Nacional de Colombia, Palmira. Colombia.

Márquez, G. 2001. De la abundancia a la escasez. La transformación de ecosistemas en Colombia. p. 1-86. En Palacios, G. (ed.). La naturaleza en disputa. Universidad Nacional de Colombia. Unibiblos. Bogotá, Colombia.

Michener, C.D. 1946. Notes on the habits of some Panamian stingless bees (Hymenoptera, Apidae). J. N. Y. Entomol. Soc. 54: 179-197.

Michener, C.D 2000. The bees of the world. The Johns Hoopkins University, Maryland, EEUU.

Nagamitsu, T. \& T. Inoue. 1997. Aggressive foreign of social bees as a mechanism of floral resource partioning in an Asian tropical rain forest. Oecologia 110: 432-439.

Nates-Parra, G. \& O. Cepeda. 1983. Comportamiento defensivo en algunas especies de meliponinos colombianos (Hymenoptera: Meliponinae). Bol Departamento de Biología Universidad Nacional de Colombia 1: 65-82.

Nates-Parra, G. 2001. Las abejas sin aguijón (Hymenoptera: Apidae: Meliponini) de Colombia. Biot. Col. 2: 233248.

Nates-Parra, G. 2005 Abejas corbiculadas de Colombia (Hymenoptera: Apidae) . Unibiblos, Universidad Nacional de Colombia, Bogotá. Colombia.

Nates-Parra, G., A. Rodríguez \& E.D. Vélez. 2006 Abejas sin aguijón (Hymenoptera: Apidae: Meliponini) en Cementerios de la Cordillera Oriental de Colombia. Acta. Biol. Col. 11: 25-35.

Nates-Parra, G. \& A. Parra-H. 2006 Uso de abejas silvestres en la definición de áreas prioritarias de conservación en el territorio CAR. Tacayá 14: 4-7. 
Parra-H, A. \& G. Nates-Parra. 2007. Variación de la comunidad de abejas de las orquídeas (Hymenoptera: Apidae) en tres ambientes perturbados del piedemonte llanero colombiano. Rev. Biol. Trop. 55: 931-941.

Rangel-Ch. J.O. \& Aguilar-P. M. 1995. Una aproximación sobre la diversidad climática en las regiones naturales de Colombia. p 25-76. En Rangel-Ch. J. O. (ed.). Colombia diversidad biótica I. Instituto de Ciencias Naturales. Universidad Nacional de Colombia. Bogotá. Colombia.

Roubik, D. 1979. Nest and colony characteristics of stingless bees from French Guiana (Hymenoptera: Apidae). J. Kans. Entomol. Soc. 52: 443-470.

Roubik, D. 1983. Nest and colony characteristics of stingless bees from Panama (Hymenoptera: Apidae). J. Kans. Entomol. Soc. 56: 327-355.

Roubik, D. 1989. Ecology and natural history of tropical bees. Cambridge University. Nueva York, EEUU.

Salmah, S., T. Inoue \& S.F. Sakagami. 1990. An analysis of apid bee richness (Apidae) in Central Sumatra, p. 139-174. En: Sakagami, S.F., R. Ohgushi \& D. Roubik (eds). Natural History of Social UASP and Bees in ecuatorial Sumatra. Hokkaido University, Hokkaido, Japón.

Sheperd, M., S.L. Buchmann, M. Vaughan \& S.H. Black. 2003. Pollinator conservation handbook. The Xerces Society. Pórtland, Oregon, EEUU.
Slaa, E.J. 2003. Foraging ecology of stingless bees: From individual behavior to community ecology. Universiteit Utrecht. The Netherlands.

Schwarz, H.F. 1932. The genus Melipona. The type genus of the Meliponidae or stingless bees. B. Am. Mus. Nat. His. 63: 231-460.

Schwarz, H. F. 1948. Stingless bees (Meliponidae) of the Western Hemisphere. B. Am. Mus. Nat. His. 90:1546.

Wille, A. \& C.D. Michener. 1973. The nest architecture of stingless bees with special reference to those of Costa Rica. Rev. Biol. Trop. 21: 1-278.

\section{REFERENCIAS DE INTERNET}

Aguilar, I. 1999 El Potencial de las Abejas Nativas Sin Aguijón (Apidae: Meliponinae) en los Sistemas Agroforestales, http:/www.cipav.org.co/redagrofor/ memorias99/AguilarI.htm. Consultado 26 mayo 2008 .

Bustamante, C. 1999 La Zonificación Ambiental como Estrategia para la Planificación de los Sistemas Agroforestales y la Recuperación y Conservación de los Recursos Naturales, http://www.cipav.org.co/ redagrofor/memorias99/BustamC.htm. Consultado 26 mayo 2008. 